\title{
Cell Therapy for Stroke
}

\author{
Sean I. Savitz,* Jonathan H. Dinsmore, ${ }^{\dagger}$ Lawrence R. Wechsler, ${ }^{\ddagger}$ Daniel M. Rosenbaum,,${ }^{\S}$ and \\ Louis R. Caplan* \\ *Department of Neurology, Beth Israel Deaconess Medical Center and Harvard Medical School, Boston, Massachusetts 02215; \\ ${ }^{\dagger}$ Department of Cell Transplantation, GenVec, Inc., Charlestown, Massachusetts 02129; ${ }^{\ddagger}$ Department of Neurology, University of \\ Pittsburgh Medical Center, Pittsburgh, Pennsylvania 15213; and ${ }^{\S}$ Departments of Neurology, Neuroscience, and Ophthalmology,
} Albert Einstein College of Medicine, Bronx, New York 10461

\begin{abstract}
Summary: Increasing experimental evidence suggests that cell transplantation can enhance recovery from stroke in animal models of focal cerebral ischemia. Clinical trials have been investigating the effects of a human immortalized neuronal cell line and porcine fetal neurons in stroke victims with persistent and stable deficits. Preclinical studies are focusing on the effects of human stem cells from various sources including brain,
\end{abstract}

bone marrow, umbilical cord, and adipose tissue. This review presents an overview of preclinical and clinical studies on cell therapy for stroke. We emphasize the current, limited knowledge about the biology of implant sources and discuss special conditions in stroke that will impact the potential success of neurotransplantation in clinical trials. Key Words: Bone marrow, porcine, stem cell, stroke, transplantation.

\section{INTRODUCTION}

Recent advances in thrombolysis and emerging successes in neuroprotective strategies have propelled acute stroke management into a therapeutic era. Once the damage from a stroke has maximized, however, little can be done to recover premorbid function. Despite immediate medical attention, many patients still have persistent deficits. Several novel "neurorestorative" approaches are being investigated as adjunctive treatments to physiotherapy. Experimental animal data supports the safety and effectiveness of cell transplantation to enhance neurological recovery from stroke. A variety of cells as implant sources have been investigated, including stem cells, immortalized cell lines, marrow and adipose stromal cells, and porcine fetal cells. Buoyed by the success of animal data, clinical trials have already commenced. This review discusses the initial clinical trials on cell therapy for stroke and their experimental animal basis. We review cell therapies in preclinical phases and point out practical and theoretical issues unique to cell transplantation for stroke.

Address correspondence and reprint requests to Sean I. Savitz, M.D., Department of Neurology, Division of Cerebrovascular Disease, Beth Israel Deaconess Medical Center, Palmer 127, Brookline, MA 02215. E-mail: ssavitz@bidmc.harvard.edu.

\section{SPECIAL CONDITIONS IN NEUROTRANSPLANTATION FOR STROKE}

Compared with neurodegenerative disease, stroke poses special conditions that impact the potential success of transplantation to enhance neurological recovery, including the anatomy and time of the stroke, the vascular supply, site of implantation, and type of patients enrolled in clinical trials.

\section{Anatomy}

In contrast to a neurodegenerative disorder such as Parkinson's disease, which destroys a relatively homogenous population of neurons, strokes affect multiple different neuronal phenotypes. For example, an infarct might involve the thalamus, hippocampus, and striate visual cortex, affecting three or more very different neuronal populations. Specific types of cells with restricted fates may limit their use as potential sources for implantation in stroke. Moreover, neurons are not the only cell type damaged. Oligodendrocytes, astrocytes, and endothelial cells are also affected. Reconstitution of the complex and widespread neuronal-glial-endothelial interrelationships may require access to a broader array of lineage species than more committed phenotypes. Cells for transplant may need to initially remain immature and phenotypically plastic to differentiate into appropriate neural, glial, and endothelial cell types depending on the 
ectopic site. Strokes also affect the white matter in addition to gray matter. If white matter is destroyed in a stroke, cell implants may not produce functional connections with axons that can penetrate through the scar tissue of a chronic infarct.

Stroke can disrupt various neuroanatomical pathways including motor, sensory, cerebellar, and visual tracts as well as the networks for attention, language, and praxis. The clinical features of stroke are, therefore, variable, whereas many of the neurodegenerative conditions cause well defined collections of impairments.

Which stroke lesions are amenable to cell transplantation? Most preclinical ischemia studies involve intrastrial implantation. Studies of the middle cerebral artery (MCA) rodent model have shown that the striatum is the primary site of damage, and many believe that the resulting deficits in memory, leaning, and motor behavior are directly associated with striatal injury. The striatum and the rest of the basal ganglia are anatomically well defined and stereotactically accessible by following a trajectory under the sylvian fissure. Cortical lesions also may be accessible to transplantation, but infarcts involving the white matter are more problematic. A proliferation of transplanted cells in the cortex may not repair underlying axonal damage. There is even less rationale for neural transplants in patients with pure white matter infarcts, which require an entirely different therapeutic strategy.

Finally, the size and extent of infarction involving major arterial territories will play a significant role in patient selection. Ideally, a limited number of cells will reasonably cover the involved area. In patients with widespread damage, however, the number of cells potentially needed to restore function may be daunting.

\section{Timing}

The appropriate time to transplant after a stroke is unknown. In the acute setting, release of excitotoxic neurotransmitters, free radicals, and proinflammatory mediators might threaten new tissue introduced into the peri-infarct region. ${ }^{1}$ Ischemic injury may also be an ongoing process. Cells may be dying by apoptosis in the penumbra for several weeks after stroke. ${ }^{2}$ Furthermore, inflammation leading to microglial activation may inhibit endogenous neurogenesis and may thereby suppress the growth and survival of transplanted cells. ${ }^{3,4}$ On the other hand, it may be better to take advantage of local repair processes, including the release of neurotrophic factors from the intrinsic milieu and the host environment during the early recovery phase to facilitate implant growth, survival, differentiation, and/or integration. The ischemic environment also promotes the generation of new neurons in periventricular regions and in the cerebral cortex. ${ }^{5,6}$ How transplantation will affect on-going endogenous neurogenesis is unknown. Delaying transplantation for weeks, however, poses the disadvantage of allowing the formation of scar tissue, which might adversely affect implanted cells.

The choice of timing must also consider the natural course of recovery from stroke. Neurodegenerative disorders are inexorably progressive and clinicians know patients will worsen over time. However, much less is known about stroke outcome, which can be quite variable. Impairments have different courses of improvement, depending on the type and severity. ${ }^{7}$ In addition, individual brains are very differently wired. For example, some patients who become aphasic after a left caudate infarct regain some language function by using their intact cerebral hemisphere, although others do not. Many neurologists would, therefore, delay transplantation until deficits have plateaued. On the other hand, there is accumulating evidence that stroke recovery involves plasticity of connections, which occur early after a stroke but may disappear months or years later. Transplantation might benefit from such plasticity and become maximally beneficial during this reorganization.

For these reasons and many others, some investigators have preferred to transplant at least a few months after a stroke. Indeed, the two clinical trials have chosen to study disabled patients at least 6 months after a stroke. Unfortunately, there are no corroborating animal models of chronic stroke to investigate transplantation several months after focal ischemia. Few outcome measures exist for animals with chronic stroke infarcts. Furthermore, functional recovery in animals cannot be easily equated across studies or related to humans.

\section{Vasculature}

Transplantation is unlikely to succeed if there is a severe arterial occlusion without collateral circulation; inadequate blood supply would not support graft survival. In the acute and subacute stages of stroke, inflammatory cells travel from the vasculature into the ischemic region. Inflammation may therefore hinder implants from taking hold in infarcted areas. In contrast, transplantation efforts in progressive degenerative disorders are not necessarily concerned with arterial patency and inflammation.

\section{Site of implant}

Does the region of the brain that receives the transplanted cells influence different responses of the donor cells? From a mechanical standpoint, injection of cells into the fluid-filled cavity of a chronic infarct facilitates the migration of transplanted cells. Without a definable, cavitated area, transplantation requires more direct pressure to inject implants, risking damage to normal tissue. However, cavity fluid can dilute the concentration of donor cells.

Many studies directly inject cells into the core of an infarct, where it remains unclear whether new tissue can remain viable. ${ }^{8}$ Specific neuropathological conditions 
TABLE 1. Various Cell Types under Investigation for Transplantation in Experimental and Clinical Stroke Trials

\begin{tabular}{|c|c|c|c|}
\hline Cell Type & Description & Potential Advantages & Potential Disadvantages \\
\hline NT2N & Immortalized cell line & Unlimited supply long-term safety & Lineage restricted \\
\hline LGE & Fetal pig & Abundant supply & $\begin{array}{l}\text { Lineage restricted } \\
\text { Pig infections }\end{array}$ \\
\hline BMSC & Bone marrow & Can be taken from patient & $\begin{array}{l}\text { Painful extraction } \\
\text { Intravenous approach may affect other organs }\end{array}$ \\
\hline HUCBC & $\begin{array}{l}\text { Umbilical cord blood } \\
\text { cells }\end{array}$ & Alternative source to bone marrow & Incomplete studies \\
\hline Adipose & Stromal stem cells & Abundant source & Incomplete studies \\
\hline NSC & Neural stem cells & Extraction from the patient & Incomplete studies \\
\hline
\end{tabular}

may alter the balance of regional environmental signals by releasing, for example, proinflammatory and other modulatory cytokines, which, in turn, may adversely affect survival and differentiation of the implanted populations. Other studies suggest that the chronic ischemic region can support implanted tissue (see below).

In the acute setting, it may be more appropriate to inject cells in the salvageable penumbra but grafts might still be exposed to the detrimental effects of spreading depression and excitatory neurotransmitters. Differences in graft behavior, depending on the injection site, were noted in a prior study. ${ }^{9}$ Fetal cortical grafts to the ischemic rat brain have been shown to survive in the penumbra but not in the core lesion. However, in chronic infarcts, glial scarring might impede the delivery of cells to the penumbral areas.

Some posit that grafts could be more effective if the poorly vascularized, inflammatory environment of the ischemic region is avoided altogether and suggest the plausibility of transplantation to distant regions, even to the contralateral side. ${ }^{10}$

For some types of transplanted cells, there may be limited regions in the ischemic brain to support their growth. Conversely, certain areas of the brain may allow some types of grafts, particularly the more multipotential and proliferative types, to grow unchecked and form tumors in contrast to regions that promote implant differentiation. $^{11}$

\section{Patients}

The selection of stroke patients for transplantation trials depends upon a number of factors. They should have measurable deficits, impairments, and handicaps. The neuroanatomical relationship between image-defined infarct and deficits should be well established. Basal ganglia strokes, for example, typically cause a hemiparesis that is easily quantifiable on neurological exam. The deficit should be sufficiently disabling to warrant this procedure but not so severe that some dramatic effect would be necessary to produce measurable improvement. Practical issues, for example, comorbidities and the need for extensive follow-up, also play a strong role in deter- mining which patients are good candidates for experimental therapies.

\section{CELL TYPES FOR STROKE NEUROTRANSPLANTATION}

A range of different cell types are under investigation (Table 1). Stem cells have been drawn from embryonic, bone marrow, and umbilical cord sources and share the advantage of being proliferative and phenotypically plastic; however, expansion in vitro to the extent needed for transplantation may be a difficult obstacle. In vitro expanded neural stem cells (NSCs) lose the capacity to differentiate. Xenotransplantation with porcine fetal cells is an alternative source, but immunosuppression and infection with endogenous pig retroviruses are potential concerns. Finally, immortalized cell lines from human tissue promises an unlimited supply and obviates the ethical concerns with stem cells. The NTera2 (NT2) cell line is the most extensively investigated in stroke transplantation. We discuss cell therapies that have advanced to clinical trials on stroke recovery, along with their animal experimental basis, and we review progress on different cell types in preclinical stages.

\section{CLINICAL TRIALS}

Initial clinical trials have exclusively enrolled patients with chronic basal ganglia infarcts who have stable and persistent deficits at least 6 months after stroke. These patients had no improvement in the months before inclusion. Two trials began in the late 1990s, one of which is on-going, the other of which was prematurely terminated. Several new trials are about to be launched.

\section{NT2 neuron cell trials}

Background. Because the widespread use of human fetal tissue is limited by logistical and ethical barriers, several laboratories have pursued efforts to develop alternative sources of tissue for transplantation. One alternative source is the immortalized cell line NT2. NT2 cells were derived from a human testicular germ cell 
tumor more than 20 years ago. ${ }^{12}$ Unlike other teratocarcinoma cell lines, the NT2 cells show an exclusive commitment to a neural lineage when exposed to retinoic acid (RA). Several studies have shown that NT2 cells resemble neural stem cells. They express cell surface markers and cytoskeletal proteins unique to neural stem cells. Exposure to RA induces the sequential expression of neural proteins in subsequent dividing progeny, recapitulating the maturational events during neurogenesis in vivo. NT2-treated cells also yield a complement of daughter cells that retain the original phenotype. ${ }^{13}$ Treatment with RA and mitotic inhibitors for several weeks ultimately results in the production of postmitotic, neuron-like cells (NT2N), ${ }^{13,14}$ which express neurotransmitters, ${ }^{15}$ functional glutamate receptors, ${ }^{16}$ calcium channels, ${ }^{17}$ mature neurofilament and cytoskeletal proteins, ${ }^{18}$ and other proteins indicative of secretory activity and synaptogenesis. ${ }^{14,19}$ They fail to acquire a fully mature phenotype in culture and terminally differentiate only when transplanted to the animal brain. ${ }^{20}$ Grafted NT2N cells to the brains of adult nude mice show evidence for terminal differentiation by 6-8 weeks and elaborate processes within 3 weeks. The cells survive up to 14 months without reverting to a neoplastic state, ${ }^{20}$ a significant concern given that these cells derive from a cancer cell line. Transplanted cells also release neurotransmitters and elaborate typical neuronal proteins.

Preclinical model. Demonstrating the survival and partial integration of grafted NT2N cells to the adult brain has led to several studies on the efficacy of transplants in rodent models of focal cerebral ischemia. Intrastriatal transplantation of NT2N cells at 1 month after MCA occlusion followed by immunosuppression with cyclosporin led to significant improvement in the passive avoidance test (a test of learning behavior retention time) and the elevated body swing test compared with ischemic animals that received rat fetal cerebellar grafts, growth medium alone, or cyclosporin alone. ${ }^{21}$ NT2N implants stained for human neuronal markers in the host striatum. As expected, grafts did not survive in nonimmunosuppressed animals. Although the authors speculated that NT2N cells might perform the functions of lost striatal cells, no studies have addressed the phenotype of NT2N implants in the transplanted ischemic brain, nor has it been shown whether they extend processes and integrate into the host brain. Aside from striatal injuries, the effects of NT2N cells in other ischemic infarcts remain to be determined.

The ready, constant availability of cryopreserved, pure neurons has made the NT2N cells an attractive implant source. Purifying neuronal NT2N cultures, however, may also limit their clinical utility. Glia are likely to be necessary for reconstruction of the infarcted cytoarchitecture and oligodendrocytes will be needed to myelinate newly formed axonal connections. Neuronal graft sur- vival may depend on glial support. It has been shown, for example, that coculture of NT2N cells with astrocytes substantially prolongs survival and enhances maturation and synaptogenesis in vitro compared with NTN2 cultures alone. ${ }^{19,22}$

Phase 1. Five years ago, a clinical trial began to assess the safety of intrastriatal NT2N (produced by Layton Bioscience, Inc. and known as LBS neurons for human use) transplantation in patients with basal ganglia infarcts and stable motor deficits 6 months to 6 years before implantation. Twelve patients were treated with NT2N cell transplants and immunosuppressed using cyclosporin for 9 weeks. Based on preclinical safety data, doses of 2 and 6 million cells were considered appropriate. Four years after the study began, there have been no adverse events related to the implants. Subsequent medical problems since transplantation have been attributed to cardiovascular risk factors and advancing age. Two patients died of unrelated medical illnesses. On autopsy examination of one of these patients, who did not show clinical improvement and died of myocardial infarction, the graft site showed no signs of inflammation, neoplasia, or infectious disease 27 months after implantation. Because NT2N cells are polypoid for chromosome 21, grafted neurons were identified at the injection site with fluorescent in situ hybridization and DNA probes specific to this distinctive chromosomal feature. ${ }^{23}$ The detection of NT2N DNA supports the contention that grafts can survive in the human brain 27 months after implantation. Positron emission tomography scanning at 6 months showed greater than $15 \%$ relative uptake of F-18 fluorodeoxyglucose at the transplant site in six patients. This may reflect surviving and functioning implanted cells, enhanced host cell activity, or an inflammatory response.

Phase 2. A randomized, open-label trial with observerblinded neurological evaluations was undertaken to test the effectiveness of neuronal cell transplantation in patients with substantial functional motor deficits following basal ganglia infarction. Fourteen patients were randomized to receive 5 or 10 million implanted cells followed by rehabilitation, compared with 4 patients who only underwent physiotherapy. Patients had stable motor deficits 1-6 years after the onset of stroke. Half the patients had an ischemic stroke, whereas the other half had a hemorrhage. The authors tested the hypothesis that implantation of neuronal cells would be safe, feasible, and improve motor neurologic deficits. One patient had a single seizure and another had a subdural hematoma evacuated 1 month after transplantation without new neurological deficits. There were no cell-associated adverse events.

Functional outcomes were assessed by the National Institutes of Health Stroke Scale, European Stroke Scale score, Stroke Impact Scale, Fugel-Meyer score, and Action Research Arm testing. Comprehensive cognitive 
testing was performed before treatment and after 6 months. Transplant patients showed a trend toward improvement in functional outcomes on several scales compared with baseline measurements before transplantation, but there were no statistically significant trends compared with the four controls. The changes were more prominent in the patients receiving 5 million cells compared with those transplanted with 10 million cells; however, with such small groups of patients, the significance of this finding is unclear. Several of the transplanted patients with non-dominant hemisphere strokes showed improvement on tests of memory, recall, and visuospacial/constructional ability on repeat testing 6 months after transplantation. The control patients did not show such changes.

Future trials. A third clinical trial will evaluate cell implantation for patients with stable cortical strokes.

\section{Diacrin trial}

Preclinical model. Given the limited availability of human tissue, some investigators have turned to fetal xenotransplants, specifically from pigs, which are considered relatively safe as a donor cell source. Transplantation of fetal cells from the porcine, primordial striatum, also called the lateral ganglionic eminence (LGE) was first shown to promote graft integration and to improve deficits in an animal model of Huntington's disease. ${ }^{24,25}$ LGE cells transplanted to the ischemic striatum 3, 7, 14, or 28 days after MCA occlusion lead to implant survival and the formation of solid grafts within the infarct cavity. Grafts differentiated into glia and neurons, some of which expressed markers for GABA and a striatal phenotype. Some porcine neurons elaborated extensive processes to host structures. There was evidence for synaptogenesis both within the graft and within the host. Animals transplanted 14 days after stroke showed statistically significant functional improvement compared with controls 4 weeks after implantation. However, no statistical differences were found at later time points. ${ }^{26}$

Despite in vivo evidence supporting the safety and viability of LGE transplants, several issues about the biology of these cells remained unresolved. LGE neurons are presumed to be undifferentiated striatal precursor cells, but no studies have investigated the extent of their development nor their potential for proliferation or differentiation. Do they differentiate into the phenotype of the ectopic site or are they committed to becoming mature striatal neurons? Studies suggest that LGE cells differentiate into striatal neurons after injection into the striatum, hippocampus, or spinal cord. ${ }^{27}$ Therefore, LGE cells at the time of donor harvest may be appropriate only for strokes involving the striatum. There have been other concerns for pig-to-human transmission of porcine endogenous retrovirus (PERV) but no studies have found evidence for PERV in patients with neurodegenerative disease who received LGE implants. ${ }^{28}$

Phase 1. A pilot safety and feasibility study was started in 1998. The original goal was to enroll 12 patients with chronic, stable, moderate-sized basal ganglia infarcts who would receive intrastriatal implantation of LGE cells. Fetal cell suspensions were prepared from dissection of the LGE of porcine embryonic tissue and pretreated in culture with an anti-major histocompatibility complex class I antibody, thus obviating the need for immunosuppression after transplantation. Five patients underwent transplantation. Their strokes occurred on average 5 years before transplantation. All patients were discharged from the hospital on the morning after surgery. Computed tomography at the completion of surgery showed no evidence of hemorrhage in any patient. The patients developed no new neurological deficits in the acute setting. However, one patient developed a cortical vein occlusion thought to be related to the surgery, not to the transplanted cells. Although the patient did not sustain any permanent damage, the study was terminated indefinitely by the Food and Drug Administration. At 2 years, none of the patients showed improvement on the modified Rankin scale. Together, the clinical trials on NT2 and LGE cells suggest that transplantation is feasible but does pose risks of seizure, subdural hematoma, and venous occlusion. It remains unclear whether implanted cells lead to any sustained long-term benefits. We await further studies to provide more definitive answers.

\section{PRECLINICAL AND PLANNED STUDIES}

In contrast to the clinical trials that are testing the biological and physiological effects of differentiated cells, most of the preclinical studies on stroke transplantation are using human, multipotential stem cells derived from the bone marrow, umbilical cord blood, or adipose tissue.

\section{Bone marrow stromal cells}

Unlike hematopoietic stem cells, bone marrow stromal cells (BMSCs) can generate a variety of tissues, including bone, cartilage, adipose, muscle, hepatocytes, glia, and neurons. ${ }^{29-31}$ When exposed to selective growth factors, human BMSCs differentiate into cells expressing markers of neural progenitors. ${ }^{29}$ Chopp and colleagues $^{32-24}$ have published a series of reports that BMSCs can ameliorate recovery after focal cerebral ischemia in rats. Direct intrastriatal, intracarotid, and intravenous delivery of BMSCs lead to migration of cells to ischemic infarcts, survival and differentiation into neuronal and glial cell types, and functional improvement. $^{32-34}$ Among 2 million BMSCs injected into the carotid artery, only $0.02 \%$ stained for neural markers in the ischemic hemisphere. The mechanisms 
of recovery are more likely due to the release of trophic factors, ${ }^{35}$ possibly promoting endogenous repair mechanisms, reducing cell death, and stimulating neurogenesis and angiogenesis ${ }^{36,37}$ rather than neuronal differentiation and implant integration to the injured ischemic site.

The success of preclinical animal data has set the stage for a limited, early-phase clinical trial using autologous BMSCs for intravenous administration to patients with stroke. Such a study would be the first step in establishing feasibility and safety of intravenous bone marrow/ mesenchymal cell transfusion for acute stroke (S. Cramer, personal communication).

There are many open-ended questions about the safety of bone marrow cells in the brain. If the transplanted cells promote recovery through the supply of trophic factors, then what ultimately happens to these cells in the brain? Do they remain in the ischemic region? Do they survive over the long term? If the cells persist, do they differentiate into different mature cell types, do they become extraneous, inert tissue versus active tumors? Long-term follow-up studies are needed to address these questions. Another issue is that only a percentage of transplanted cells administered intravenously migrate to the ischemic area. The rest spread to other areas of the brain and to other organs, where they could potentially change organ physiology and promote the formation of ectopic tumors. Homing of bone marrow stem cells to sites of organ injury, including the ischemic brain, is believed to occur through a complex multistep process. Chemotactic factors are likely responsible for attracting transplanted cells to the ischemic regions of the brain, ${ }^{38}$ but other routes of administration, such as an intracarotid approach, may be necessary to minimize spread to other tissues.

\section{Human umbilical cord stem cells}

The umbilical cord is another source of multipotential stem cells that, when exposed to selective growth factors, create progeny that stain for neuronal and glial cell markers. ${ }^{39}$ Even less is understood about the biology of human umbilical cord blood cells (HUCBCs). Nevertheless, a study has already shown that intravenous infusion of these undifferentiated cells to rats $24 \mathrm{~h}$ after MCA occlusion improved behavioral recovery 14 days after implantation compared with control animals subjected to focal ischemia alone or focal ischemia with saline injection. ${ }^{40}$ Approximately $1 \%$ of these cells survived and migrated to the ischemic hemisphere, where an estimated $2 \%$ expressed neuronal markers. As expected, intravenously administered HUCBCs also spread to various other organs. A head-to-head trial comparing intravenous versus intrastriatal injection of HUCBCs $24 \mathrm{~h}$ after MCA occlusion showed similar degrees of improvement with one functional test favoring the intravenous transplants at
2 months after transplant; however, transplanted HUCBCs could not be detected in the host brain of rats that received cyclosporine, a troubling result that has not adequately been explained. ${ }^{41}$ Similar to BMSCs, the functional effects of HUCBCs in the ischemic brain observed within such a short period of time likely result from release of trophic factors.

\section{Human adipose stromal cells}

Recent studies also suggest that adipose tissue contains pluripotent stromal cells. Primary cultures of adipose tissue are a heterogenous collection of hematopoietic cells, pericytes, endothelial cells, and smooth muscle cells. Several passages in cultures yields stromal cells that exhibit cell-surface markers consistent with mesenchymal stem cells ${ }^{42,43}$; they express nestin, suggesting the potential for differentiation into neural lineage species, but do not express markers for hematopoietic stem cells or macrophages. ${ }^{43}$ Stromal cells cultured from human liposuction adipose tissue can be directed toward neural differentiation with 5-azacytidine/NGF/BDNF/ bFGF and the resulting progeny express MAP2 and GFAP. ${ }^{43}$ The ability to direct stromal cells from adipose tissue along neural lineages suggests that they may have potential therapeutic applications for neuronal disorders.

Adipose tissue is therefore being investigated as a potential autoplastic therapy for stroke. Injection of human adipose tissue stromal cells into the lateral ventricle of healthy rats results in migration to multiple areas of the brain, including the contralateral cortex, and the cells persist at their destination at 30 days after implantation. Injection to the lateral ventricle 1 day after MCA occlusion led to migration into the ischemic area where implanted cells were visualized at the border between intact and injured brain, but cells also traveled far distances including the contralateral cortex. At 7 days after stroke, transplanted rats had significantly better recovery in motor and somatosensory behavior compared with animals that only received saline. There were no differences in infarct size between the two groups. ${ }^{43}$ The long-term behavior and effects of these cells in the brain are unknown, but the temporal course of benefit strongly argues against engraftment and integration into the host structure and continues to support current hypotheses that transplanted stem cells after stroke provide trophic support.

\section{Human neural stem cells}

Neural cells with stem cell properties have been isolated from the embryonic, neonatal, and adult rodent brain and propagated in culture by growth factors, mitogens, and immortalizing genes. Several laboratories have also prepared stem cell lines from primary dissociated cell cultures of embryonic human CNS. Their effects on the ischemic adult animal brain have not been studied to the same extent as stem cells from other tissues. One of 
these cell lines, retrovirally transduced with the v-myc oncogene, has been studied in a rat model of intraparenchymal hemorrhage. Intravenous administration $24 \mathrm{~h}$ after the induction of intracranial hemorrhage led to improved motor performance 2 weeks later. Transplanted cells had also been infected with a retroviral vector containing lac $\mathrm{z}$ to permit detection in vivo and were found in both the perihematomal region and the contralateral hemisphere, some of which stained for glial and neuronal markers. ${ }^{44}$ Although there may have been a benefit observed from transplantation, the biological behavior of these cells in the hemorrhagic brain requires additional extensive investigation. It is also important to keep in mind that this hemorrhage model is an entirely different pathology than ischemic stroke, fraying axonal connections but not destroying neuronal cell bodies.

In a neonatal hypoxic-ischemic model, an immortalized, neural stem cell line derived from the murine cerebellar external granular layer was transplanted into injured rat brains and cells were detected up to 22 months after engraftment. Initial results showed that $5 \%$ of engrafted NSCs in the injured hemisphere differentiated into neurons, whereas there were no new neurons in the contralateral, intact hemisphere. ${ }^{45}$

Using an immortalized stem cell line from the hippocampal proliferative zone of the transgenic mouse (MHP36 cells), investigators at ReNeuron have shown that transplanted cells reduced infarct volumes and improved sensorimotor recovery but not spatial learning or memory over the 18 weeks that followed MCA occlusion. Cells were injected into the intact somatosensory cortex and striatum, contralateral to the infarct cavity and an estimated $30 \%$ of the transplanted cells migrated to the lesioned cortex and striatum. ${ }^{10}$ The plasticity of this cell line remains unclear but it has been shown that MHP36 cells in this model showed diverse phenotypes of different neuron and glia-like morphologies. ${ }^{10}$ Current efforts in several laboratories and companies are now directed at studying a range of human, genetically engineered neural stem cell lines in animal models of stroke.

\section{FUTURE OF STROKE NEUROTRANSPLANTATION}

Future work in the field of stroke neurotransplantation needs to focus on several issues. Among them, we discuss five concerns related to stroke. First, what is the intended strategy for implantation? In neurodegenerative disease such as Parkinson's disease, the goal is to reconstitute a dopaminergic neural network. In animal models of stroke, transplanted cells may play more a role as neurotrophin "pumps" rather than as grafted tissue restoring segmental connections. The mechanism of recovery might impact the choice of cells to implant. If a trophic response is desired, then a cell line genetically engineered to supply trophic support may be a better choice. If reconstitution of a neural network is desired, then access to a broader array of lineage species may be necessary to reconstruct the complex and widespread neuronal, glial, and endothelial damage in stroke. This point leads to our second concern; it is crucial to better understand the basic biological mechanisms of individual cell types as implant sources for transplantation in stroke patients. Such properties that need further clarification are proliferation, differentiation, potential lineage restrictions, and extent of integration into host structures

Our third concern is safety. Long-term studies on the biological behavior of cell grafts are mandatory to better understand the effects and safety of cell transplantation in the infarcted brain. Parallel studies in the heart have demonstrated that stem cell-derived cardiomyocytes show spontaneous activity and easily inducible triggered arrhythmias. ${ }^{46}$ Thus, transplanted cells to the heart could be arrythmogenic. Similarly, transplanted stem cells in the brain ultimately may prove to be epileptogenic. Furthermore, there remains virtually no information regarding the long-term effects on organ physiology and tumor formation from parenteral delivery of transplanted cells. Methods may need to be developed to target the ischemic brain while minimizing spread to other tissues.

Fourth, the number of cells needed to promote recovery is a matter of debate. This issue impacts delivery methods given the volumes needed for adequate cell number. The numbers needed could exceed practical limits depending on the type of implant.

Fifth, how does transplantation modulate the response of the brain to ischemic injury including endogenous neurogenesis, synaptogenesis, angiogenesis, and inflammation? Inducing de novo neurogenesis may prove a more effective and safer therapeutic strategy to promote recovery from stroke rather than transplanting exogenous cells. ${ }^{47}$ An attractive, yet purely speculative future goal may be the extraction of a stroke patient's own neural stem cells, expansion and support in vitro, and reimplantation to the ischemic brain.

\section{CONCLUSIONS}

Basic and clinical research in stroke neurotransplantation remains in a nascent stage. Much more work is needed to further characterize the biology of different implant sources both in vitro and in vivo. Initial clinical data suggests that transplantation is technically feasible and can be performed safely, but the data are too preliminary and insufficient to assess efficacy.

Acknowledgments: We thank Dr. Michael Alexander for helpful comments about stroke recovery. L.R.W. receives grant funding from Layton Bioscience. GenVec, Inc. funded the porcine transplant trial at the Beth Israel Deaconess Medical Center. 


\section{REFERENCES}

1. Lo EH, Dalkara T, Moskowitz MA. Mechanisms, challenges and opportunities in stroke. Nat Rev Neurosci 4:399-415, 2003.

2. Li Y, Chopp M, Jiang N, Yao F, Zaloga C. Temporal profile of in situ DNA fragmentation after transient middle cerebral artery occlusion in the rat. J Cereb Blood Flow Metab 15:389-397, 1995.

3. Ekdahl CT, Claasen JH, Bonde S, Kokaia Z, Lindvall O. Inflammation is detrimental for neurogenesis in adult brain. Proc Natl Acad Sci USA 100:13632-13637, 2003.

4. Li Y, Chen J, Chopp M. Cell proliferation and differentiation from ependymal, subependymal and choroid plexus cells in response to stroke in rats. J Neurol Sci 193:137-146, 2002

5. Nakatomi H, Kuriu T, Okabe S et al. Regeneration of hippocampal pyramidal neurons after ischemic brain injury by recruitment of endogenous neural progenitors. Cell 110:429-441, 2002.

6. Arvidsson A, Collin T, Kirik D, Kokaia Z, Lindvall O. Neuronal replacement from endogenous precursors in the adult brain after stroke. Nat Med 8:963-970, 2002.

7. Hier DB, Mondlock J, Caplan LR. Recovery of behavioral abnormalities after right hemisphere stroke. Neurology 33:345-350, 1983.

8. Grabowski M, Johansson BB, Brundin P. Neocortical grafts placed in the infarcted brain of adult rats: few or no efferent fibers grow from transplant to host. Exp Neurol 134:273-276, 1995.

9. Hadani M, Freeman T, Munsiff A, Young W, Flamm E. Fetal cortical cells survive in focal cerebral infarct after permanent occlusion of the middle cerebral artery in adult rats. J Neurotrauma 9:107-112, 1992.

10. Veizovic T, Beech JS, Stroemer RP, Watson WP, Hodges H. Resolution of stroke deficits following contralateral grafts of conditionally immortal neuroepithelial stem cells. Stroke 32:10121019, 2001.

11. Miyazono M, Lee VM, Trojanowski JQ. Proliferation, cell death, and neuronal differentiation in transplanted human embryonal carcinoma (NTera2) cells depend on the graft site in nude and severe combined immunodeficient mice. Lab Invest 73:273-283, 1995.

12. Andrews PW, Damjanov I, Simon D et al. Pluripotent embryonal carcinoma clones derived from the human teratocarcinoma cell line Tera-2. Differentiation in vivo and in vitro. Lab Invest 50:147-162, 1984.

13. Pleasure SJ, Lee VM. NTera 2 cells: a human cell line which displays characteristics expected of a human committed neuronal progenitor cell. J Neurosci Res 35:585-602, 1993.

14. Pleasure SJ, Page C, Lee VM. Pure, postmitotic, polarized human neurons derived from NTera 2 cells provide a system for expressing exogenous proteins in terminally differentiated neurons. $\mathrm{J} \mathrm{Neu-}$ rosci 12:1802-1815, 1992.

15. Guillemain I, Alonso G, Patey G, Privat A, Chaudieu I. Human NT2 neurons express a large variety of neurotransmission phenotypes in vitro. J Comp Neurol 422:380-395, 2000.

16. Younkin DP, Tang CM, Hardy M et al. Inducible expression of neuronal glutamate receptor channels in the NT2 human cell line. Proc Natl Acad Sci USA 90:2174-2178, 1993.

17. Neelands TR, King AP, Macdonald RL. Functional expression of L-, N-, P/Q-, and R-type calcium channels in the human NT2-N cell line. J Neurophysiol 84:2933-2944, 2000.

18. Trojanowski JQ, Mantione JR, Lee JH et al. Neurons derived from a human teratocarcinoma cell line establish molecular and structural polarity following transplantation into the rodent brain. Exp Neurol 122:283-294, 1993.

19. Hartley RS, Margulis M, Fishman PS, Lee VM, Tang CM. Functional synapses are formed between human NTera2 (NT2N, hNT) neurons grown on astrocytes. J Comp Neurol 407:1-10, 1999.

20. Kleppner SR, Robinson KA, Trojanowski JQ, Lee VM. Transplanted human neurons derived from a teratocarcinoma cell line (NTera-2) mature, integrate, and survive for over 1 year in the nude mouse brain. J Comp Neurol 357:618-632, 1995.

21. Borlongan CV, Tajima Y, Trojanowski JQ, Lee VM, Sanberg PR. Transplantation of cryopreserved human embryonal carcinoma- derived neurons (NT2N cells) promotes functional recovery in ischemic rats. Exp Neurol 149:310-321, 1998.

22. Bani-Yaghoub M, Felker JM, Naus CC. Human NT2/D1 cells differentiate into functional astrocytes. NeuroReport 10:3843-3846, 1999.

23. Nelson PT, Kondziolka D, Wechsler L et al. Clonal human (hNT) neuron grafts for stroke therapy: neuropathology in a patient 27 months after implantation. Am J Pathol 160:12011206, 2002.

24. Isacson O, Deacon TW, Pakzaban P, Galpern WR, Dinsmore J, Burns LH. Transplanted xenogeneic neural cells in neurodegenerative disease models exhibit remarkable axonal target specificity and distinct growth patterns of glial and axonal fibres. Nat Med 1:1189-1194, 1995.

25. Deacon TW, Pakzaban P, Burns LH, Dinsmore J, Isacson O. Cytoarchitectonic development, axon-glia relationships, and long distance axon growth of porcine striatal xenografts in rats. Exp Neurol 130:151-167, 1994.

26. Dinsmore JH, Martin J, Siegan J, Morrison JP, Lindberg C, Ratliff J, Jacoby D. CNS grafts for treatment of neurologic disorders. In: Methods of tissue engineering, Ed 1, pp 1127-1134. San Diego: Academic Press, 2002.

27. Jacoby DB, Lindberg C, Cunningham MG, Ratliff J, Dinsmore J. Long-term survival of fetal porcine lateral ganglionic eminence cells in the hippocampus of rats. $J$ Neurosci Res 56:581594, 1999.

28. Dinsmore JH, Manhart C, Raineri R, Jacoby DB, Moore A. No evidence for infection of human cells with porcine endogenous retrovirus (PERV) after exposure to porcine fetal neuronal cells. Transplantation 70:1382-1389, 2000.

29. Sanchez-Ramos J, Song S, Cardozo-Pelaez F et al. Adult bone marrow stromal cells differentiate into neural cells in vitro. Exp Neurol 164:247-256, 2000.

30. Prockop DJ. Marrow stromal cells as stem cells for nonhematopoietic tissues. Science 276:71-74, 1997.

31. Woodbury D, Schwarz EJ, Prockop DJ, Black IB. Adult rat and human bone marrow stromal cells differentiate into neurons. J Neurosci Res 61:364-370, 2000.

32. Li Y, Chopp M, Chen J et al. Intrastriatal transplantation of bone marrow nonhematopoietic cells improves functional recovery after stroke in adult mice. J Cereb Blood Flow Metab 20:1311-1319, 2000.

33. Li Y, Chen J, Wang L, Lu M, Chopp M. Treatment of stroke in rat with intracarotid administration of marrow stromal cells. Neurology 56:1666-1672, 2001.

34. Chen J, Li Y, Wang L et al. Therapeutic benefit of intravenous administration of bone marrow stromal cells after cerebral ischemia in rats. Stroke 32:1005-1011, 2001.

35. Chen $\mathrm{X}, \mathrm{Li} \mathrm{Y}$, Wang $\mathrm{L}$ et al. Ischemic rat brain extracts induce human marrow stromal cell growth factor production. Neuropathology 22:275-279, 2002.

36. Chen J, Zhang ZG, Li Y et al. Intravenous administration of human bone marrow stromal cells induces angiogenesis in the ischemic boundary zone after stroke in rats. Circ Res 92:692699, 2003.

37. Chen $\mathrm{J}, \mathrm{Li} \mathrm{Y}$, Katakowski $\mathrm{M}$ et al. Intravenous bone marrow stromal cell therapy reduces apoptosis and promotes endogenous cell proliferation after stroke in female rat. J Neurosci Res 73:778786, 2003.

38. Hill WD, Hess DC, Martin-Studdard A et al. SDF-1 (CXCL12) is upregulated in the ischemic penumbra following stroke: association with bone marrow cell homing to injury. $J$ Neuropathol Exp Neurol 63:84-96, 2004.

39. Sanchez-Ramos JR, Song S, Kamath SG et al. Expression of neural markers in human umbilical cord blood. Exp Neurol 171:109-115, 2001.

40. Chen J, Sanberg PR, Li Y et al. Intravenous administration of human umbilical cord blood reduces behavioral deficits after stroke in rats. Stroke 32:2682-2688, 2001. 
41. Willing AE, Lixian J, Milliken M et al. Intravenous versus intrastriatal cord blood administration in a rodent model of stroke. J Neurosci Res 73:296-307, 2003.

42. Gronthos S, Franklin DM, Leddy HA, Robey PG, Storms RW, Gimble JM. Surface protein characterization of human adipose tissue-derived stromal cells. J Cell Physiol 189:54-63, 2001.

43. Kang SK, Lee DH, Bae YC, Kim HK, Baik SY, Jung JS. Improvement of neurological deficits by intracerebral transplantation of human adipose tissue-derived stromal cells after cerebral ischemia in rats. Exp Neurol 183:355-366, 2003.

44. Jeong SW, Chu K, Jung KH, Kim SU, Kim M, Roh JK. Human neural stem cell transplantation promotes functional recovery in rats with experimental intracerebral hemorrhage. Stroke 34:22582263, 2003.

45. Park KI. Transplantation of neural stem cells: cellular and gene therapy for hypoxic-ischemic brain injury. Yonsei Med J 41:825835, 2000.

46. Zhang YM, Hartzell C, Narlow M, Dudley SC Jr. Stem cellderived cardiomyocytes demonstrate arrhythmic potential. Circulation 106:1294-1299, 2002.

47. Abrahams JM, Gokhan S, Flamm ES, Mehler MF. De novo neurogenesis and acute stroke: are exogenous stem cells really necessary? Neurosurgery 54:150-156, 2004. 